Revista Digital Universitaria

Vol. 23, Núm. 1, enero-febrero 2022

\title{
Blockchain en la educación: su uso en credenciales académicas
}

\author{
José Miguel Mata Hernández y Sandra Avendaño Cruz
}

\begin{abstract}
Resumen
A través de la tecnología de la cadena de bloques (blockchain) es posible solucionar problemas como la emisión, la agilización y la realización de acreditaciones académicas de una forma íntegra, práctica y eficiente. La aplicación de esta tecnología en la educación y por parte de universidades generaría acreditaciones académicas donde el estudiante sea dueño de su credencial y que ésta sea verificable, única, infalsificable, portable, descargable y emitida en poco tiempo.
\end{abstract}

Palabras clave: cadena de bloques, credenciales académicas, acreditaciones académicas, educación.

\section{BLOCKCHAIN IN EDUCATION: ITS USE IN ACADEMIC CREDENTIALS}

\begin{abstract}
Through the technology of blockchain, problems such as issuing, streamlining, and carrying out academic accreditations can be solved in a complete, practical, and efficient way. The application of this technology in education and by universities would generate academic accreditations where the student is the owner of his academic credential and that this is verifiable, unique, unfalsifiable, portable, downloadable, and issued in a short time.
\end{abstract}

Keywords: blockchain, academic credentials, academic accreditations, education. 
José Miguel Mata Hernández

josemiguelmata@hotmail.com orcid.org/0000-0003-0132-6881

LinkedIn: miguel-mata-hern

Posgrado de la Facultad de Contaduría y Administración de la Universidad Nacional Autónoma de México (UNAM). Licenciado en Negocios y Comercio Internacional por la Universidad Autónoma de Querétaro. Especialidad en Gestión de Riesgos Financieros y Empresariales EUDE Business School. Especialidad en tráfico y tramitación de mercancías ETTA-EscI. Maestría en Finanzas Bursátiles por el Posgrado de la Facultad de Contaduría y Administración, unAm. Escritor colaborador en Rankia México. Sus investigaciones están basadas en temas relacionados con el tipo de cambio, trading, Fintech, criptomonedas, pymes, economía y finanzas.

\title{
Sandra Avendaño Cruz
}

\author{
sandyuamyunam@gmail.com \\ orcid.org/0000-0002-2888-6136 \\ LinkedIn: sandra-avenda
}

Posgrado de la Facultad de Contaduría y Administración de la unAm. Licenciada en Economía por la fEs Aragón, unAM. Maestría en Finanzas Corporativas por el Posgrado de la Facultad de Contaduría y Administración, UNAM. Jefa de Unidad en Comisión Nacional del Sistema de Ahorro para el Retiro. Sus investigaciones están basadas en temas relacionados con el tipo de cambio, índice sustentable, econometría, economía y finanzas. 
"Blockchain en la educación: su uso en credenciales académicas" José Miguel Mata Hernández y Sandra Avendaño Cruz Vol. 23, Núm. 1, enero-febrero 2022

Revista Digital Universitaria

\section{Introducción}

$\mathrm{E}$ I proceso que conlleva la acreditación académica consiste en varios pasos que toman bastante tiempo. Este proceso puede durar semanas o hasta meses en llevarse a cabo y entregado al solicitante. La acreditación académica sirve para demostrar que la persona estudió un grado, un curso o un programa. Esto se demuestra a través de la credencial o certificado emitido por una institución o universidad; sin embargo, ésta puede llegar a ser falsificada. En cuanto a los problemas con los que se enfrenta un solicitante con su acreditación académica están los relacionados con el tiempo de emisión, la comprobación de su autenticidad y la validez.

Se entiende a la tecnología blockchain como aquella tecnología descentralizada que registra las operaciones efectuadas de una forma inalterable, a través de un consenso que las legitima, por lo que está distribuida entre muchas partes que son las que la validan. Uno de los beneficios de su uso es que las operaciones realizadas dentro del blochckain son verificables y no se pueden borrar o modificar, lo que brinda certeza, integridad y confianza (Bit2Me Academy, s. f.).

La importancia del uso del blockchain radica en que las instituciones educativas la pueden utilizar para poder enfrentar las problemáticas del tiempo de emisión y de la realización de la acreditación académica, para que ésta sea demostrable, registrable e inmutable ${ }^{1}$. Por lo tanto, dichas acreditaciones estarían resguardadas en una blockchain, con lo cual se brindaría certeza, seguridad, integridad y confianza al emitirlas (Han et al., 2018).

El desarrollo y mejora de esta tecnología y su aplicación en el ámbito de la educación ha aumentado su uso por parte de las universidades, lo cual ha sido beneficioso, ya que ha permitido la agilización de los procesos de emisión de las acreditaciones académicas. Blockchain cuenta con la capacidad de transformar muchos sistemas tradicionales a una forma más segura, completa, distribuida y transparente. Asimismo, añade protección al mantenimiento de las credenciales de los estudiantes y evita la falsificación de las acreditaciones académicas (Matthews, 2017).

La implementación de esta tecnología crea una infraestructura para documentar, almacenar y gestionar las acreditaciones académicas; por lo que llega a proporcionar a los alumnos un registro sostenible de sus logros que ellos mismos pueden controlar, de acuerdo con Jirgensons y Kapenieks (2018).

\section{Plataformas que sustentan credenciales blockchain}

Las insignias digitales conocidas como digital badges son credenciales digitales que demuestran las habilidades y logros que llegan a ser adquiridos en algún programa o curso. Fueron creadas con el fin de representar los conocimientos
${ }^{1}$ La inmutabilidad se refiere a que no se pueden modificar 
adquiridos para que fueran demostrables y que se pudieran compartir con las personas interesadas, con el fin de exponer los logros y los conocimientos obtenidos. Estas insignias sirvieron de base para la implementación de la tecnología blockchain en las acreditaciones académicas; es por ello por lo que plataformas como Accredible pueden emitir este tipo de insignias con tal tecnología.

Las acreditaciones académicas en blockchain cuentan con ventajas en comparación con las acreditaciones académicas tradicionales. De acuerdo con Álvarez (2021), éstas son:

1. Permiten al estudiante tener control sobre sus acreditaciones académicas.

2. Están validadas y reconocidas por la institución académica que las emite, por lo que demuestran el nivel académico obtenido.

3. Son inmutables, abiertas y transparentes porque su registro es único y cualquier persona puede verificar su validez.

4. Son seguras y previenen el fraude porque no se pueden falsificar.

5. Son portables, recuperables y se pueden descargar en diversos formatos de documento.

De las plataformas existentes dentro del mercado de acreditaciones académicas digitales en blockchain, se destacan las siguientes:

- Blockcerts por parte del Instituto Tecnológico de Massachussets, desarrollada a través de mit Media Lab utiliza la cadena de bloques de Bitcoin y de Ethereum para el registro de las acreditaciones académicas de sus estudiantes.

- EkoLink utiliza la Eko blockchain, una cadena de bloques con enfoque empresarial, con el objetivo de crear acreditaciones académicas digitales, ésta misma es compatible con la blockchain de Ethereum.

- Accredible ofrece el servicio de registrar acreditaciones académicas a través de la blockchain de Bitcoin.

- Open Blockchain a través del Knowledge Media Institute por parte de la Universidad Abierta del Reino Unido, utiliza la blockchain de Ethereum para el registro de las acreditaciones académicas de sus estudiantes.

- Block.Co, por parte del Instituto para el Futuro de la Universidad de Nicosia de Chipre, utiliza la blockchain de Bitcoin para el registro de las acreditaciones académicas de los programas, cursos y títulos de grado de sus estudiantes.

En la figura 1 se pueden observar las plataformas de los proyectos que utilizan la tecnología de la cadena de bloques para la emisión de acreditaciones académicas. 
Figura 1. Plataformas que utilizan blockchain para credenciales educativas.
${ }^{2}$ Las cadenas de bloques públicas son aquellas donde el registro es visible por todos los usuarios en la red.

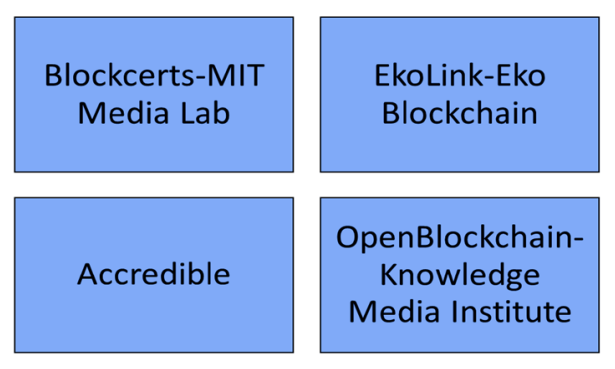

\section{Funcionamiento de Blockcerts y Block.co}

Las entidades que se dedican a ofrecer acreditaciones académicas bajo esta tecnología usualmente hacen uso de una cadena de bloques pública², que puede ser la de Bitcoin o la de Ethereum. Existen dos plataformas destacadas en cuanto a su funcionalidad: Blockcerts y Block.co; ambas utilizan la cadena de bloques de Bitcoin.

En Blockcerts:

- Las acreditaciones académicas no pueden ser falsificadas y pueden ser verificadas dentro de la plataforma. En el caso de que haya algún error dentro de la credencial (Ej. Que el nombre esté mal), no será posible hacerle modificaciones. La persona tendrá que pedir que se anule y después solicitar una nueva.

- La entidad emisora utiliza su firma digital para brindar una credencial a un receptor que es la persona solicitante; ésta es identificada por una clave pública que es propiedad del solicitante, y transmitida en la Blockchain. Para que sea procesada la acreditación-credencial académica dentro de la cadena de bloques de Bitcoin, es necesario que se realice la prueba de Merkle, la cual consiste en resumir de una manera efectiva todas las transacciones del bloque dentro de un formato compacto, esto con el fin de establecer la integridad de la credencial.

- Las credenciales con la clave pública de identificación de propiedad de la persona solicitante sirven como un ID único de identificación.

\section{En Block.co:}

- En el caso de las credenciales emitidas, se les puede plasmar información de lo visto en un programa o grado o curso, es decir, el certificado emitido puede tener información de los logros o habilidades obtenidas. Asimismo, se muestra información sobre en que blockchain fue emitida la acreditación académica, la identificación del emisor y la identificación de la transacción donde se resguardó la acreditación académica.

Para observar cómo se resguarda una acreditación académica dentro de la cadena de bloques de Bitcoin, se comparte el siguiente enlace:

https://blockstream.info/tx/669cec5e2b262b5e382d78c5653e7fdc5 e929da1cf091256fce5bf6e6316deed 
"Blockchain en la educación: su uso en credenciales académicas" José Miguel Mata Hernández y Sandra Avendaño Cruz Vol. 23, Núm. 1, enero-febrero 2022

Revista Digital Universitaria

Asimismo, para ejemplificar como luce un certificado resguardado dentro de la cadena de bloques de Bitcoin con el uso de Bloc.co, se comparte el siguiente enlace:

\section{https://app.block.co/certificate/b3db4ccf-0e0b-45c9-96d2-} $682 \mathrm{c} 1 \mathrm{~d} 18 \mathrm{e} 1 \mathrm{~d} 8 /$

El procedimiento para que una acreditación académica en Blockcerts sea resguardada en la cadena de bloques de Bitcoin, conlleva la realización de transacciones de entrada y de salida, es decir, se debe realizar una transacción de Bitcoin para cada lote de certificados que desean ser resguardados en la blockchain de Bitcoin. De acuerdo con el funcionamiento de una cadena de bloques, toda transacción está contenida en bloques, los cuales son validados para aceptar la autenticidad de la transacción. La parte de entrada conlleva la transacción emitida en Bitcoin, mientras que en la parte de salida se hace el almacenamiento de los certificados, donde se ocupa el código OP_RETURN, desarrollado en el código abierto Bitcoin Core con licencia MIT. Este código es ocupado con el fin de realizar la validación de los certificados. El costo de emitir un lote en Blockcerts está influenciado en gran medida por la tarifa de transacción, que es la que se paga a los mineros ${ }^{3}$ de Bitcoin por el uso de su red.

Universidades e institutos utilizan Blockcerts debido a su funcionalidad y rapidez. Esto requiere que la institución emisora de las credenciales tenga varias acreditaciones académicas a validar para solicitar a Blockcerts la creación de los certificados en la blockchain de Bitcoin, con todo el proceso que conlleva. Una vez creado el certificado, la persona que lo solicitó puede checarlo a través de la plataforma de Blockcerts y descargarlo si así lo desea. La persona solicitante de una credencial en blockchain puede descargar la aplicación de Blockcerts en su móvil e iniciar el proceso de emisión de su certificado, (véase figura 2).

Figura 2. Proceso de emisión de un certificado en Blockchain a través de Blockcerts.

Fuente. Elaboración propia con datos de Blockcerts (2021).

\footnotetext{
${ }^{3}$ Los mineros de Bitcoin son los encargados de descifrar los bloques e incorporarlos a la Red de Bitcoin, por lo que hacer uso de la red conlleva hacer el pago
} de una comisión.

\begin{tabular}{|c|c|c|c|c|}
\hline $\begin{array}{l}\text { Emisor invita al } \\
\text { solicitante para } \\
\text { recibir una } \\
\text { credencial en } \\
\text { Blockchain }\end{array}$ & $\begin{array}{l}\text { El solicitante } \\
\text { acepta la } \\
\text { invitación y envía } \\
\text { su dirección de } \\
\text { Blockchain al } \\
\text { emisor }\end{array}$ & $\begin{array}{c}\text { El emisor realiza } \\
\text { la generación de } \\
\text { nuevas } \\
\text { direcciones y } \\
\text { claves para la } \\
\text { credencial dentro }\end{array}$ & $\begin{array}{l}\text { El emisor envía al } \\
\text { solicitante su } \\
\text { credencial } \\
\text { Blockchain }\end{array}$ & $\begin{array}{c}\text { El solicitante } \\
\text { recibe su } \\
\text { credencial y la } \\
\text { puede verificar o } \\
\text { descargar o } \\
\text { compartir }\end{array}$ \\
\hline
\end{tabular}

En el caso de Block.co, el cual es muy similar al de Blockcerts, es necesario que se tenga un lote de certificados de estudiantes a emitir para realizar el proceso.

La aplicación de Blockcerts funciona como una cartera digital en donde se guardan los certificados en blockchain. Esta misma cartera se llega a conectar con otras instituciones que pueden emitir acreditaciones académicas con esta misma plataforma, por lo cual se pueden tener certificados de distintas instituciones. La contraseña o passphrase es la clave que permite el acceso a las credenciales dentro de la plataforma y la cual demuestra que se es poseedor de las credenciales dentro de la plataforma.

La cartera de Blockcerts es una de las opciones más accesibles para los estudiantes, para almacenar y gestionar sus credenciales digitales (MIT Media Lab, 2016). 
"Blockchain en la educación: su uso en credenciales académicas" José Miguel Mata Hernández y Sandra Avendaño Cruz Vol. 23, Núm. 1, enero-febrero 2022

Revista Digital Universitaria

Como afirma Schmidt (2015), la tecnología blockchain y la criptografía proporcionan una infraestructura técnica que permite el almacenamiento, la seguridad, la gestión y distribución de acreditaciones académicas digitales. Esto pone a los usuarios en un punto de control de sus logros sin necesidad de recurrir a terceros. Además, la criptografía protege la información de los estudiantes a través de cifrados, por lo que el uso de ésta en Blockcerts y en Block.co permite resguardar de forma segura la información de los estudiantes.

\section{Instituciones académicas y la tecnología blockchain}

Distintas universidades han observado el potencial de la cadena de bloques en el ámbito educativo y han desarrollado diversos proyectos. Es posible por parte de una universidad o instituto desarrollar la infraestructura para emitir la creación de acreditaciones académicas en blockchain, o en su caso contratar los servicios de terceros como lo son Blockcerts o Block.co.

Algunos casos donde se han implementado este tipo de acreditaciones académicas son:

- The Open University of United Kingdom: a través del Knowledge Media Institute desarrolló el proyecto Open Blockchain donde se realizan investigaciones sobre las aplicaciones que tiene esta tecnología, además del desarrollo de las credenciales académicas a través de la red de Ethereum.

- La Universidad de Nicosia de Chipre a través del Instituto para el futuro UNIC, emite sus acreditaciones académicas bajo la tecnología blockchain, de Bitcoin por medio de la plataforma Block.co.

- El Massachussets Institute of Technology registra los títulos académicos de sus estudiantes con tecnología la Blockchain de Bitcoin y Ethereum. Estos son verificables a través de MIT Degree Verification. Además de esto, la institución cuenta con el MIT Media Lab creadora de Blockcerts, así como el Cryptoeconomics Lab.

- El Tecnológico de Monterrey a través de su centro de investigación Mostla, realiza la emisión de certificados con tecnología blockchain por medio de la plataforma Blockcerts.

No solamente se puede hacer uso de esta tecnología blockchain en el área educativa. Como lo comenta Pina et al., (2017) su uso puede ser aplicada en otros ámbitos de la educación, como la identidad y registro de los estudiantes, la creación de nuevas formas de pedagogía a través de una sociedad digital del conocimiento, financiamiento y apoyo a estudiantes. La creación de una MetaUniversidad, es decir un Metaverso universitario.

El fomento a la investigación en la tecnología de la cadena de bloques ha sido apoyado por las fundaciones de Ethereum, de Ripple, de Cardano, donde 
a través de éstas, se han promovido investigaciones y proyectos en diferentes universidades alrededor del mundo (Getso y Johari, 2017).

\section{Conclusiones}

Las acreditaciones académicas que usan tecnología blockchain traen facilidades y beneficios tanto al estudiante como a la institución académica, porque su emisión es más rápida, práctica, segura, íntegra, verificable y confiable.

Aquellos estudiantes que tienen sus acreditaciones académicas en alguna de las plataformas que emite credenciales en blockchain, son dueños de sus credenciales, lo que les permite que éstas se puedan imprimir, enviar y compartir en cualquier momento.

Diversas universidades e instituciones académicas alrededor del mundo han empezado a emitir sus títulos, diplomas y certificados bajo esta tecnología, y ocupan los servicios de las diversas plataformas existentes comentadas en la presente investigación.

La implementación de este tipo de acreditaciones académicas bajo blockchain, sería un gran esfuerzo por parte de universidades y otras instituciones académicas en México y del mundo, por lo que su ejecución traería reducciones en cuanto a costos administrativos y cuestiones burocráticas; asimismo agilizaría la emisión de éstas y cada estudiante podría tener la custodia de sus propias acreditaciones académicas, reduciendo así también el nivel de falsificaciones. Entre los beneficios de esta tecnología se encuentran los siguientes: simplificación de procesos, ahorro en la verificación de datos e identidad y reducción en tiempo de trámites de dichas credenciales y de la autentificación.

\section{Referencias}

* Accredible. (s. f.). About Accredible. https://www.accredible.com/about

* Álvarez Marañón, G. (4 de mayo de 2021). El futuro de las credenciales universitarias apunta hacia Blockchain y Open Badges. https://cutt.ly/sU9heeg

* Blockcerts. (s. f.). About Blockcerts. https://www.blockcerts.org/about.html

* Block Co. (s. f.). About Block Co. https://block.co/about/

* Bit2Me Academy. (s.f.). ¿Qué es la Cadena de Bloques (Blockchain)? https://cutt.ly/zU9kYHH

* EchoLink. (s. f.). Introduction. https://cutt.ly/6U8uqm1

* Getso, M. M. A., y Johari, Z. (2017, abril). The blockchain revolution and higher education. International Journal of Information System and Engineering, 5(1), 57-66. https://doi.org/hb9r 
* Han, M., Li, Z., He, J., Wu, D., Xie, Y., y Baba, A. (2018). A novel blockchain-based education records verification solution. In Proceedings of the 19th Annual SIG Conference on Information Technology Education (pp. 178-183). https://doi. org/10.1145/3241815.3241870

* Jirgensons, M., and Kapenieks, J. (2018). Blockchain and the future of digital learning credential assessment and management. Journal of teacher education for sustainability, 20(1), 145-156. https://eric.ed.gov/?id=EJ1218203

* Massachusetts Institute of Technology (24 de mayo del 2021). MIT Degree Verification. https://credentials.mit.edu/

* Matthews, D. (31 de agosto de 2021). What Blockchain Technology could mean for Universities, Times Higher Education World Rankings. https://www. timeshighereducation.com/news/what-blockchain-technology-could-mean-foruniversities

* MIT Media Lab. (2 de junio de 2016). What we learned from designing an academic certificates system on the blockchain. https://medium.com/mit-media-lab/whatwe-learned-fromdesigning-an-academic-certificates-system-on-the-blockchain$34 \mathrm{ba5} 874 \mathrm{f196}$

* Mostla. (22 de mayo del 2021). Títulos electrónicos en Blockchain. https://mostla.tec. mx/titulos-blockchain/index.html

* Open Blockchain. (24 de mayo de 2021). About Blockchain Overview. https:// blockchain.open.ac.uk/about/

* Pina, A. R. B., Torlà, C. B., Quintero, L. C., y Segura, J. A. (2017). Blockchain en Educación: introducción y crítica al estado de la cuestión. Edutec. Revista Electrónica de Tecnología Educativa, (61), a363-a363.https://doi.org/10.21556/ edutec.2017.61.915

* Schmidt, P. (2015). Certificates, reputation, and the blockchain. Medium. https:// medium.com/mit-media-lab/certificatesreputation-and-the-blockchainaee03622426f

\section{Cómo CITAR ESTE ARTículo}

* Mata Hernández, José Miguel y Avendaño Cruz, Sandra. (2022, enero-febrero). Blockchain en la educación: su uso en credenciales académicas. Revista Digital Universitaria (RDU), 23(1). http://doi.org/10.22201/cuaieed.16076079e.2022.23.1.1 